\title{
N94-14990
}

\section{Ring Lens Focusing and Push-Pull Tracking Scheme for Optical Disk Systems}

\author{
R. Gerber, J. Zambuto, J.K. Erwin, and M. Mansuripur \\ Optical Sciences Center, University of Arizona, Tucson, Arizona 8572I
}

\begin{abstract}
An experimental comparison of the ring lens and the astigmatic techniques of generating focus-errorsignal (FES) in optical disk systems reveals that the ring lens generates a FES over two times steeper than that produced by the astigmat. Partly due to this large slope and, in part, because of its diffraction-limited behavior, the ring lens scheme exhibits superior performance characteristics. In particular the undesirable signal known as "feedthrough" (induced on the FES by track-crossings during the seek operation) is lower by a factor of six compared to that observed with the astigmatic method. The ring lens is easy to align and has reasonable tolerance for positioning errors.
\end{abstract}


Introduction : The ring lens technique of focus error detection and its combination with push-pull tracking was proposed in an earlier paper [1]. Later, based on diffraction calculations, the ring lens was predicted to have superior characteristics over other methods of focus-error detection [2,3]. This superiority stems from the fact that the ring lens produces a large, yet diffraction-limited image at the detector, has circular symmetry which simplifies alignment procedures, and allows focus- and track-error signals as well as data signals to be detected in the same channel. This paper reports the experimental verification of some of the above predictions; in particular, we report that the ring lens produces a steeper FES and has significantly lower feedthrough (i.e., focus error signal generated when moving across tracks) than the astigmatic technique. In the sections that follow we describe a method that has been used successfully to fabricate the ring lens, describe our static testbed for making measurements of the focus- and track-error signals, present the results of these measurements for a ring lens, and compare the results with those obtained for the astigmatic scheme.

Ring Lens Fabrication: The ring lens used in these experiments was formed on a soda-lime glass slide by a thermal process. Initially, a base plate of polished stainless steel with a circular groove carved on its flat surface was prepared. This plate was coated with a thin layer of black ink which serves as mold release agent, and then placed in contact with the glass slide. The assembly was heated in an oven to $735^{\circ} \mathrm{C}$ for 2 minutes, then slowly cooled back to the room temperature. During heating, the softened glass sagged into the groove and formed a curved surface in the annulus defined by the groove. The glass slide was subsequently removed from the mold, cleaned, and without further polishing was used in the experiments.

Figure 1 shows various features of the fabricated ring lens. Dimensions of the lens are depicted in Fig.1(a) as follows : inner diameter $=2.03 \mathrm{~mm}$, outer diameter $=4.35 \mathrm{~mm}$, focused ring radius $=$ $1.595 \mathrm{~mm}$. The unshaded annular region in the figure corresponds to the section of the lens that has been profiled by the WYKO Corporation's TOPO system and shown in Fig.1(b). A scanning electron micrograph of a section of the ring lens appears in (c), and an interferogram showing Newton rings for this lens is reproduced in (d).

It must be emphasized that although the thermal process described above yields reasonably good ring lenses, we do not consider it suitable for the fabrication of highly accurate, aberration-free lenses. Other shortcomings of this technique include the inability to produce lenses with very small ring radii, and also lack of any direct control over the focal length of the lens. Ideally, such lenses must be made by direct molding of glass or plastic, or by the fabrication methods of holographic optics or binary optics.

Testbed for Measurements of Focus/Track Error Signals : The system used to test the various focusing techniques is the static focusing/tracking testbed shown in Fig. 2. This system operates at two $\mathrm{HeNe}$ wavelengths (633 $\mathrm{nm}$ and $544 \mathrm{~nm}$ ), and allows either of two linear polarizations (parallel 
and perpendicular to tracks) to be used for measurements. The paths of the red and green beams are coincident after their combination by the dichroic beamsplitter. The beams are then split into two beams of orthogonal polarizations and recombined by the polarizing beamsplitter. One can readily select a wavelength and a polarization direction by blocking the undesired paths on the testbed. The beam intensity may be controlled by a variable attenuator placed after the dichroic splitter.

After passing through a spatial filter and a collimating lens, the beam goes through a removable shear plate collimation tester, which can detect wavefront errors as low as $0.1 \lambda$. The beam emerging from the lens is fairly large in cross-section, and is stopped by a $5 \mathrm{~mm}$ diameter aperture. The collimated beam passes through a 50/50 beam-splitter cube which sends half of the light to the disk (or an immitation of the disk) and the other half to a plane mirror, which acts as the reference arm in a Twyman-Green interferometer. The reference arm is used to check the collimation of the beam returning from the disk, and is covered during focus/track error measurements.

In our experiments the grooved surface of an optical disk was simulated by a reflection diffraction grating, placed at the focal plane of a $40 \times, 0.55 \mathrm{NA}$, infinity-corrected microscope objective. The grating has $1.1 \mu \mathrm{m}$ track-pitch, a sinusoidal profile, and peak-to-valley depth of $67 \mathrm{~nm}$. The choice of grating instead of an actual grooved disk eliminates the problem of focusing through the substrate, which would unnecessarily complicate the measurements by introducing aberrations due to misalignment and birefringence. The use of grating also affords us more flexibility in selecting the numerical aperture of the objectives, which are generally not available in a wide variety for $1.2 \mathrm{~mm}$ cover slides. The position of the grating is controlled by low-voltage piezoelectric transducers (PZT), mounted in the actuators of an XYZ translation stage. A GPIB-controlled power supply can move the PZT's in 0.003 micron increments over a range of 12 microns.

The focusing/tracking optics are placed after the beam-splitter cube, and form an image on a CCD camera whose pixel spacing is $10 \mu \mathrm{m}$. The ring lens forms an image directly on the camera, while the spot formed by the astigmat is small and requires magnification. The image from the camera is fed into a frame-grabber card, which can simulate arbitrarily-shaped detectors in software. The simulated detector for the ring lens scheme is a phi detector (so named because of its resemblance to the Greek letter $\Phi$ ), while that for the astigmatic method is a quad detector.

Focus-Error Detection and Push-Pull Tracking with the Ring Lens : The ring lens used in our experiments had a focal length of $25 \mathrm{~mm}$, and focused a collimated beam onto a sharp, nearly diffraction-limited ring of diameter $3.19 \mathrm{~mm}$. A section of the focused ring is shown in Fig. 3, as an intensity plot of the light pattern collected by the camera; the width of this ring is roughly $50 \mu \mathrm{m}$.

A schematic diagram of the ring lens focusing technique is shown in Fig. 4. As the disk (grating) moves in and out of focus, the beam incident on the ring lens becomes divergent or convergent, and the light at the focal plane of the ring lens moves outside or inside its nominal radius. A focus error signal may thus be generated by subtracting the integrated light intensity outside the nominal ring 
radius from that inside. The difference signal is subsequently normalized by the sum signal in order to eliminate the effects of laser power and/or reflectivity fluctuations.

During seek operations when the beam scans across the tracks while remaining in focus, diffraction effects produce a so-called "baseball pattern" on the returning beam. This pattern produces bright and dark regions on the focused ring. A track-crossing signal can thus be generated by subtracting the integrated intensity in one half of the image from that in the other half; again the difference signal should be normalized by the sum for reasons that were described earlier.

Focus-Error Detection and Push-Pull Tracking with the Astigmatic Lens: The astigmatic scheme is depicted in Fig. 5. The astigmat used in these experiments had focal lengths $19.75 \mathrm{~mm}$ and $20.25 \mathrm{~mm}$, and a clear aperture of about $5 \mathrm{~mm}$. The detector was placed at the circle of least confusion, midway between the two line foci, where deviations of the incident beam from collimation produced an elongated image in one of two mutually orthogonal directions. The elongation of the spot is detected by a four-quadrant detector; the FES and TES signals are generated by subtracting the integrated intensity on one pair of quadrants from that on the other, then dividing by the sum signal. Since in our experiments the spot itself was too small to be properly resolved by the CCD array, the plane midway between the foci of the astigmat was magnified by a $40 \times$ magnifier and imaged onto the camera. All subsequent processing of the signals was carried out on this magnified image.

Results and Discussion: The ring lens technique was found to produce a steeper FES curve and significantly less feedthrough than the astigmatic method. These findings apply to both wavelengths and both polarization directions employed in this work. The ring lens was also found to be fairly insensitive to misalignments.

Figures $6(a, b)$ show the FES curves for the ring lens and astigmatic techniques, respectively. The four curves in each group are for different combinations of wavelength and polarization. Neither polarization nor the wavelength seem to have a significant effect on the shape and slope of the FES curve. The slope of the linear region of the FES for the ring lens is 0.5 per micron of defocus, whereas that for the astigmat is only 0.22 per micron.

Plots of TES and spurious FES during track-crossings (i.e., feedthrough) are shown in Fig. 7 for the ring lens and Fig. 8 for the astigmat; important features of these plots are also summarized in Table I. Note that the TES amplitudes are comparable for the two schemes. On the other hand, for both wavelengths and polarizations, the feedthrough (in microns) for the ring lens is between six and eight times lower than that for the astigmat.

The ring lens technique is fairly insensitive to misalignments and imperfections of the system. Figure $9(a)$ shows several FES curves for various radii of the phi detector. (Recall that the radius of the focused ring is around $1.6 \mathrm{~mm}$, and its width is approximately $50 \mu \mathrm{m}$.) An error of $\pm 10 \mu \mathrm{m}$ in 
detector radius will change the zero-crossing by \pm 1.6 microns. In actual drive, if the radius of the detector happens to deviate from that of the focused ring, the electronic gains of the detectors must be adjusted to offset this error. Figure $9(a)$ also shows that a mismatch of $\pm 20 \mu \mathrm{m}$ between the ring radius and the radius of detector can seriously degrade the FES slope. In Fig. $9(b)$ we show FES curves for the best radius but various locations of the phi detector in the focal plane of the ring lens. These plots show a slight decrease in the slope as well as a small shift of the zero-crossing for $10 \mu \mathrm{m}$ of centering error. The consequences of a $20 \mu \mathrm{m}$ decenter, however, are much more drastic.

\begin{tabular}{|c|c|c|c|c|c|c|}
\hline \multirow{2}{*}{$\begin{array}{l}\text { Focusing } \\
\text { Scheme }\end{array}$} & \multirow{2}{*}{$\begin{array}{c}\text { Wavelength } \\
(\mathrm{nm})\end{array}$} & \multirow{2}{*}{$\begin{array}{c}\text { Polarization } \\
\text { (relative to tracks) }\end{array}$} & \multirow{2}{*}{$\begin{array}{l}\text { Peak-to-valley TES } \\
\text { norm. units }\end{array}$} & \multicolumn{3}{|c|}{ Peak-to-valley feedthrough } \\
\hline & & & & norm. units & microns & wavelengths \\
\hline Ring lens & 544 & parallel & 1.9 & 0.12 & 0.25 & 0.46 \\
\hline Ring lens & 544 & perpendicular & 1.9 & 0.12 & 0.25 & 0.46 \\
\hline Ring lens & 633 & parallel & 1.5 & 0.07 & 0.14 & 0.23 \\
\hline Ring lens & 633 & perpendicular & 1.5 & 0.08 & 0.17 & 0.27 \\
\hline Astigmat & 544 & parallel & 1.6 & 0.34 & 1.5 & 2.75 \\
\hline Astigmat & 544 & perpendicular & 1.7 & 0.44 & 2.0 & 3.65 \\
\hline Astigmat & 633 & parallel & 1.4 & 0.22 & 1.0 & 1.60 \\
\hline Astigmat & 633 & perpendicular & 1.4 & 0.22 & 1.0 & 1.60 \\
\hline
\end{tabular}

\section{Table I. Summary of track-crossing and feedthrough signals}

In comparison with the ring lens results in Fig. $9(b)$, Fig. 10 shows several FES curves for various locations of the quad detector of the astigmatic technique. We observe that $6 \mu \mathrm{m}$ of centering error has negligible effects on the FES curve, but a decenter of $12 \mu \mathrm{m}$ is beginning to cause severe problems.

Concluding Remarks : We have presented a comparison of the ring lens and astigmatic methods of generating focus- and track-error signals for optical disk drives. It was found that the ring lens produces an FES curve which is substantially steeper than that produced by the astigmat. Also the cross-talk on the focus error signal from track-crossings was 6-8 times smaller for the ring lens method. In addition, we found the ring lens scheme to be tolerant of small errors in detector positioning and alignment.

Acknowledgements : Thanks are due to Dr. B.E. Bernacki for helpful advice and many valuable discussions. We also thank Mr. J. Corso for his help in fabricating the ring lenses. 


\section{References}

1. M. Mansuripur and C. Pons, "Diffraction modeling of optical path for magneto-optical disk systems," Proceedings of the Optical Data Storage Conference, D.B. Carlin, Y. Tsunoda and A.A. Jamberdino, Eds., SPIE Vol. 899, pp56-60 (1988).

2. B.E. Bernacki and M. Mansuripur, "Diffraction Analysis and evaluation of several focus- and track-error detection schemes for magneto-optical disk systems," Proceedings of the Optical Data Storage Conference, D.B. Carlin and D.B. Kay, Eds., SPIE Vol. 1663, pp150-156 (1992).

3. B.E. Bernacki, "Characterization of magneto-optical media and systems," PhD dissertation, Optical Sciences Center, University of Arizona (1992). 


\section{Figure Captions}

Fig. 1. a) Diagram showing dimensions of the ring lens. The apex of the lens occurs at a radius of $1.595 \mathrm{~mm}$, which corresponds to the focused ring radius of a collimated incident beam.

b) Surface profile of section of the lens obtained by interferometric measurements. The region captured by the profilometer is the unshaded annular region depicted in (a) with a width of $0.524 \mathrm{~mm}$.

c) SEM image of section of the ring lens.

d) Newton's rings observed on a section of the ring lens.

Fig. 2. Schematic diagram of the static focusing/tracking testbed.

Fig. 3. Section of the intensity profile of the focused ring (pixel $=10 \mu \mathrm{m} \times 10 \mu \mathrm{m}$ ).

Fig. 4. Schematic diagram of the method of ring lens focusing and push-pull tracking.

Fig. 5. Schematic diagram of the method of astigmatic focusing and push-pull tracking.

Fig. 6. Measured FES curves for the ring lens (a) and astigmatic (b) scheme of focus error detection. The various symbols in each plot correspond to different wavelengths and polarization orientations used in the experiment.

Fig. 7. Measured TES (dashed) and feedthrough (solid) curves for the ring lens scheme.
a) Green laser with polarization parallel to tracks,
b) Green laser with polarization perpendicular to tracks,
c) Red laser with polarization parallel to tracks,
d) Red laser with polarization perpendicular to tracks.

Fig. 8. Measured TES (dashed) and feedthrough (solid) curves for the astigmatic scheme.
a) Green laser with polarization parallel to tracks,
b) Green laser with polarization perpendicular to tracks,
c) Red laser with polarization parallel to tracks,
d) Red laser with polarization perpendicular to tracks. 
Fig. 9. FES curves for the ring lens scheme in the case of red laser with polarization parallel to tracks.
a) Different curves correspond to different detector radii. The solid curve is obtained for best radius, " + " is for $\pm 10 \mu \mathrm{m}$ and " $x$ " for $\pm 20 \mu \mathrm{m}$ change in the radius. The curves shift to the left with increasing detector radius.
b) Different curves correspond to various detector locations within the focal plane of the ring lens. The solid curve ("o") is for the best detector position, while the dashed curves are for translations of $10 \mu m("+)$ and $20 \mu m(" x ")$ relative to this optimum position.

Fig.10. FES curves for the astigmatic scheme in the case of red laser with polarization parallel to tracks; different curves correspond to various detector locations within the midplane between the two astigmatic line foci. The solid curve (" $\mathrm{o}$ ") is for the best detector position, while the dashed curves are for translations of $6 \mu \mathrm{m}("+)$ and $12 \mu \mathrm{m}(" x ")$ relative to this optimum position. 



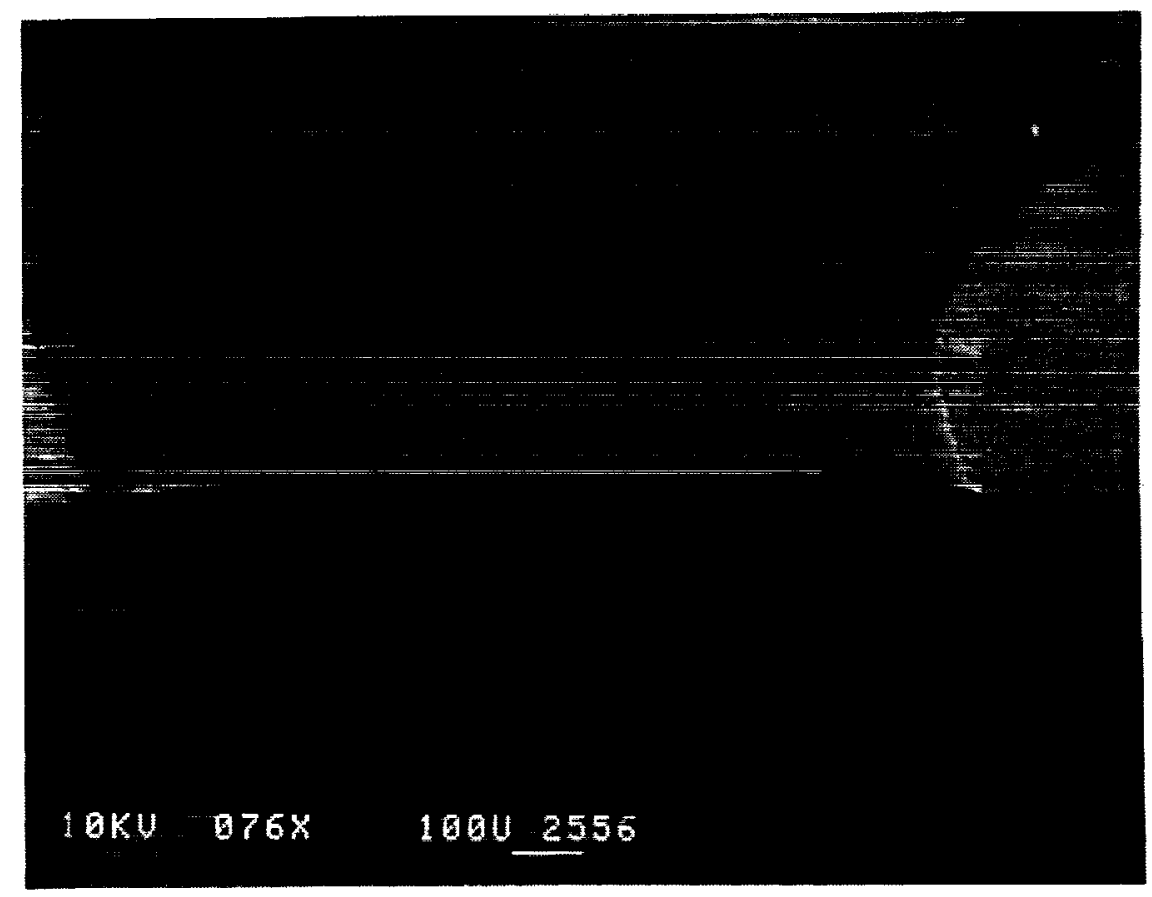

Figure 1(c)

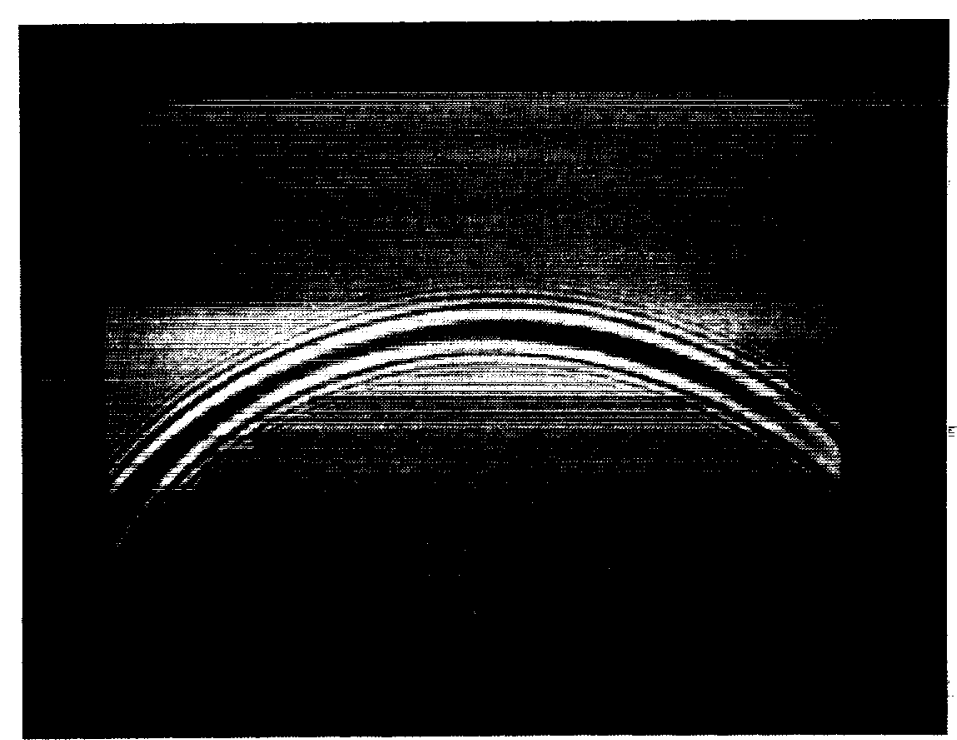

-

Figure 1(d) 



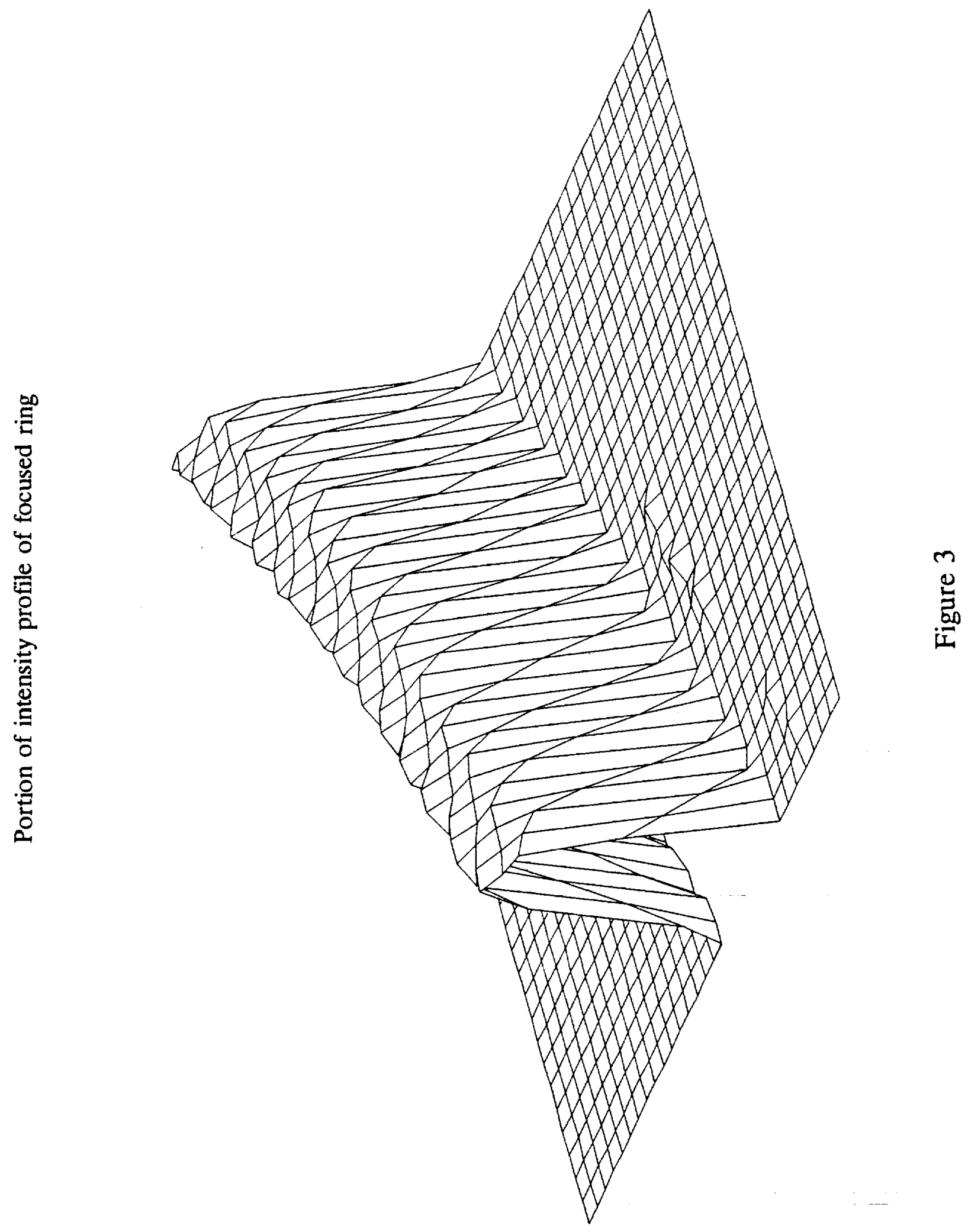




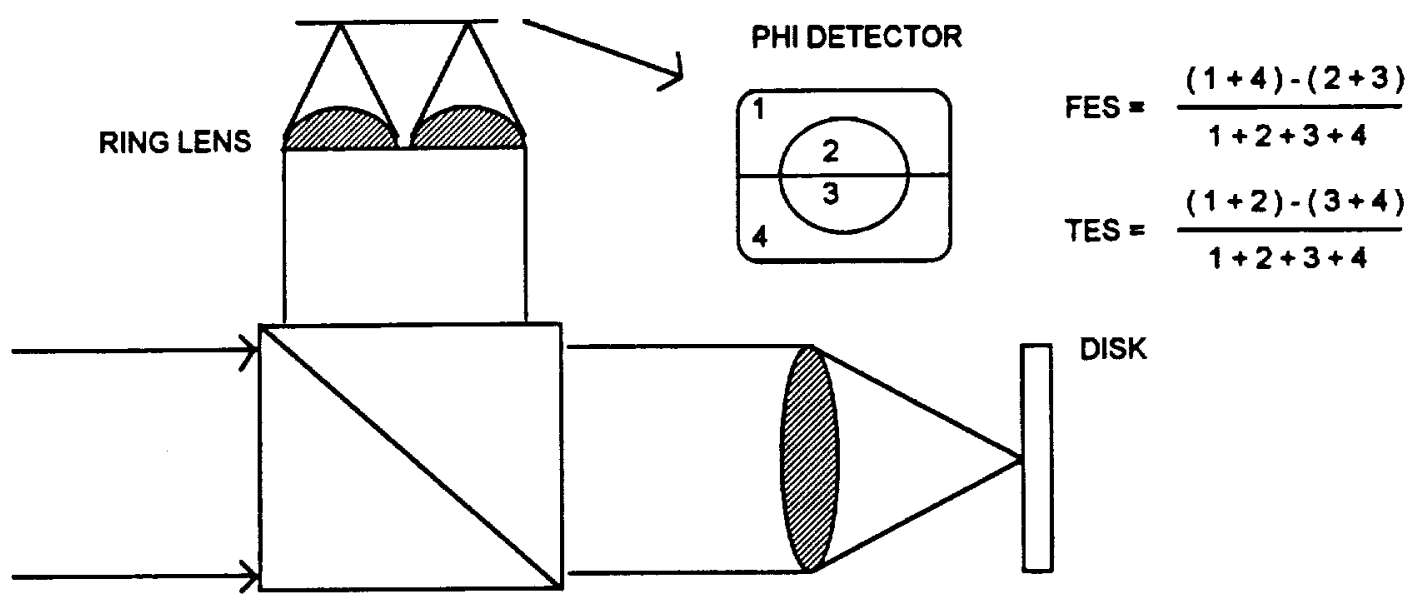

Figure 4 


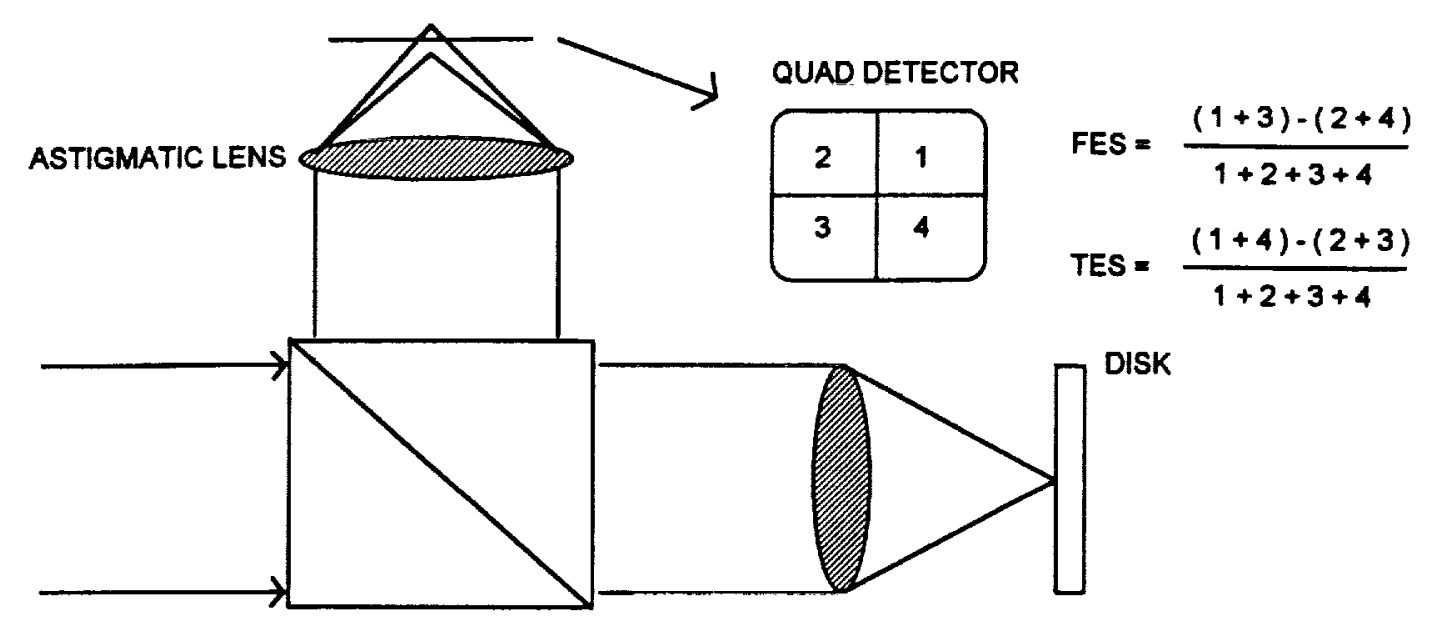

Figure 5 


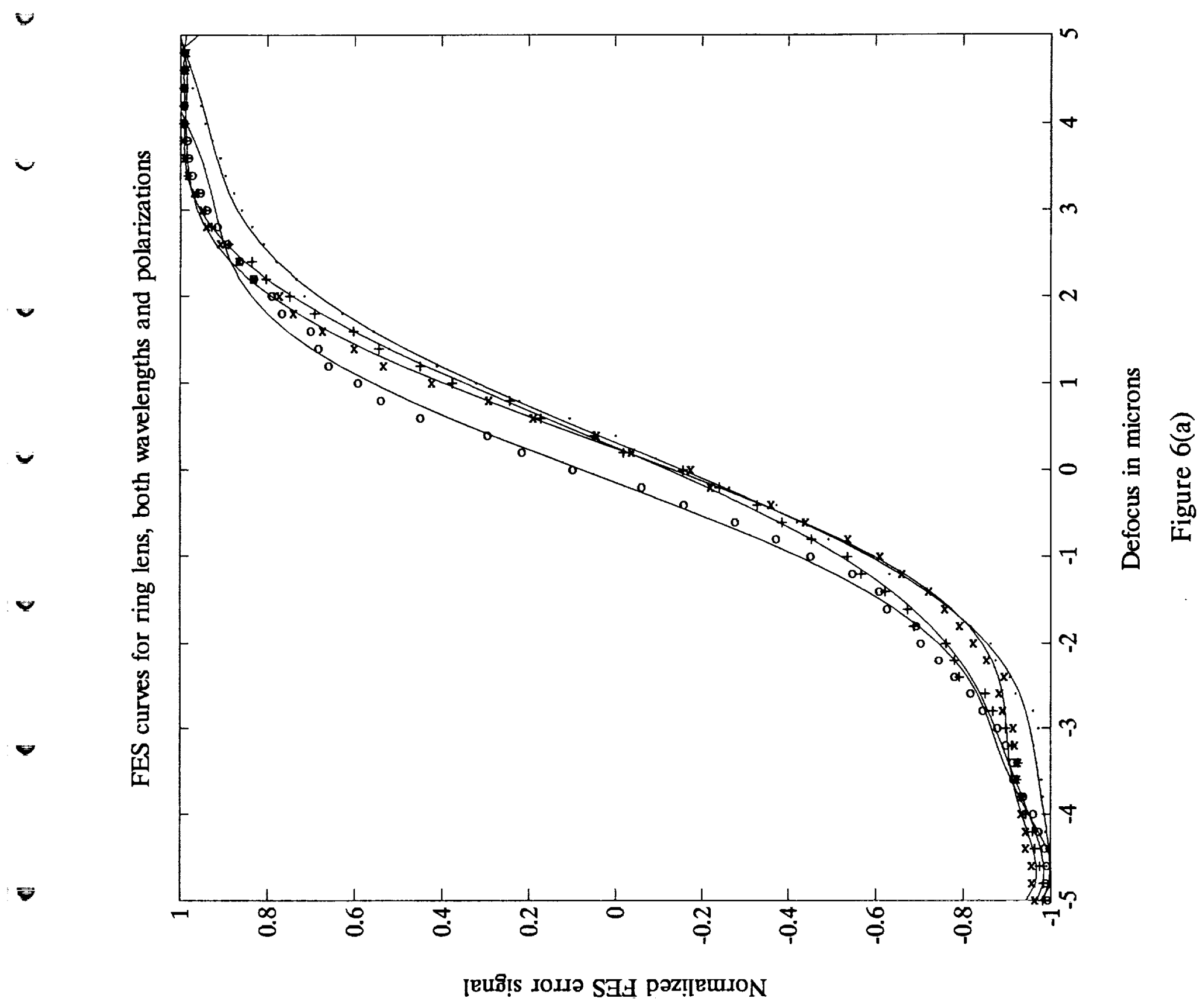




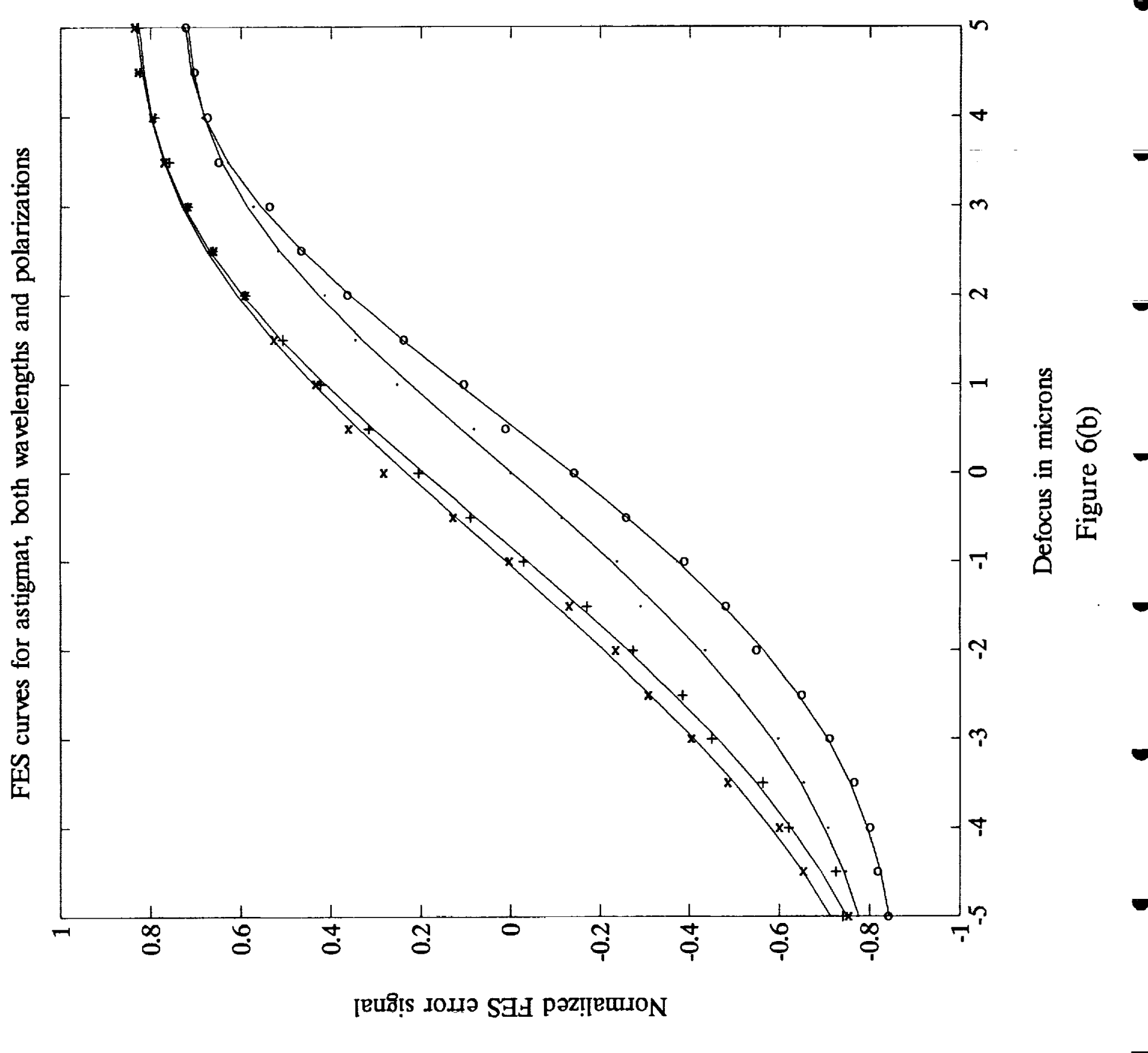




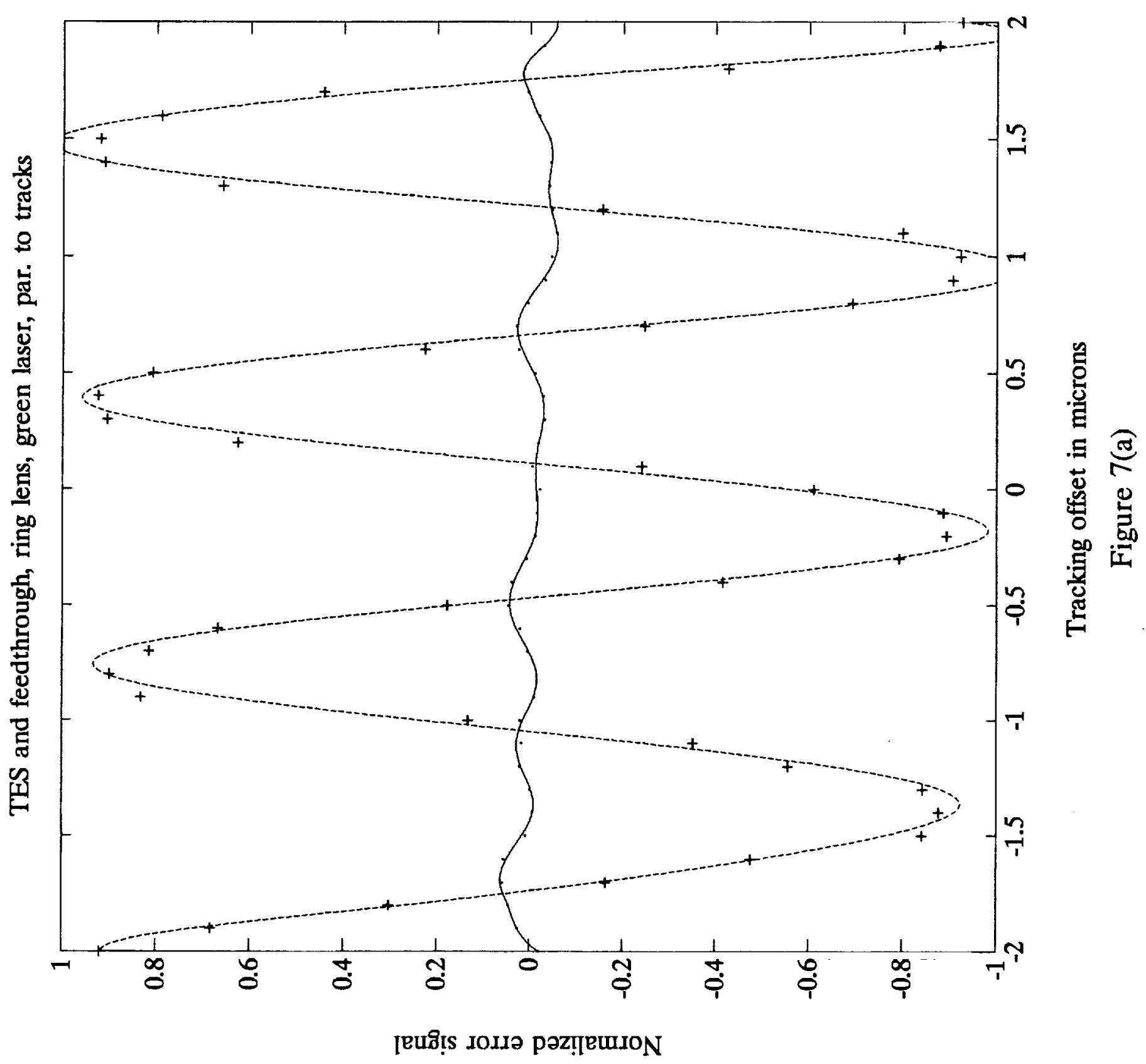




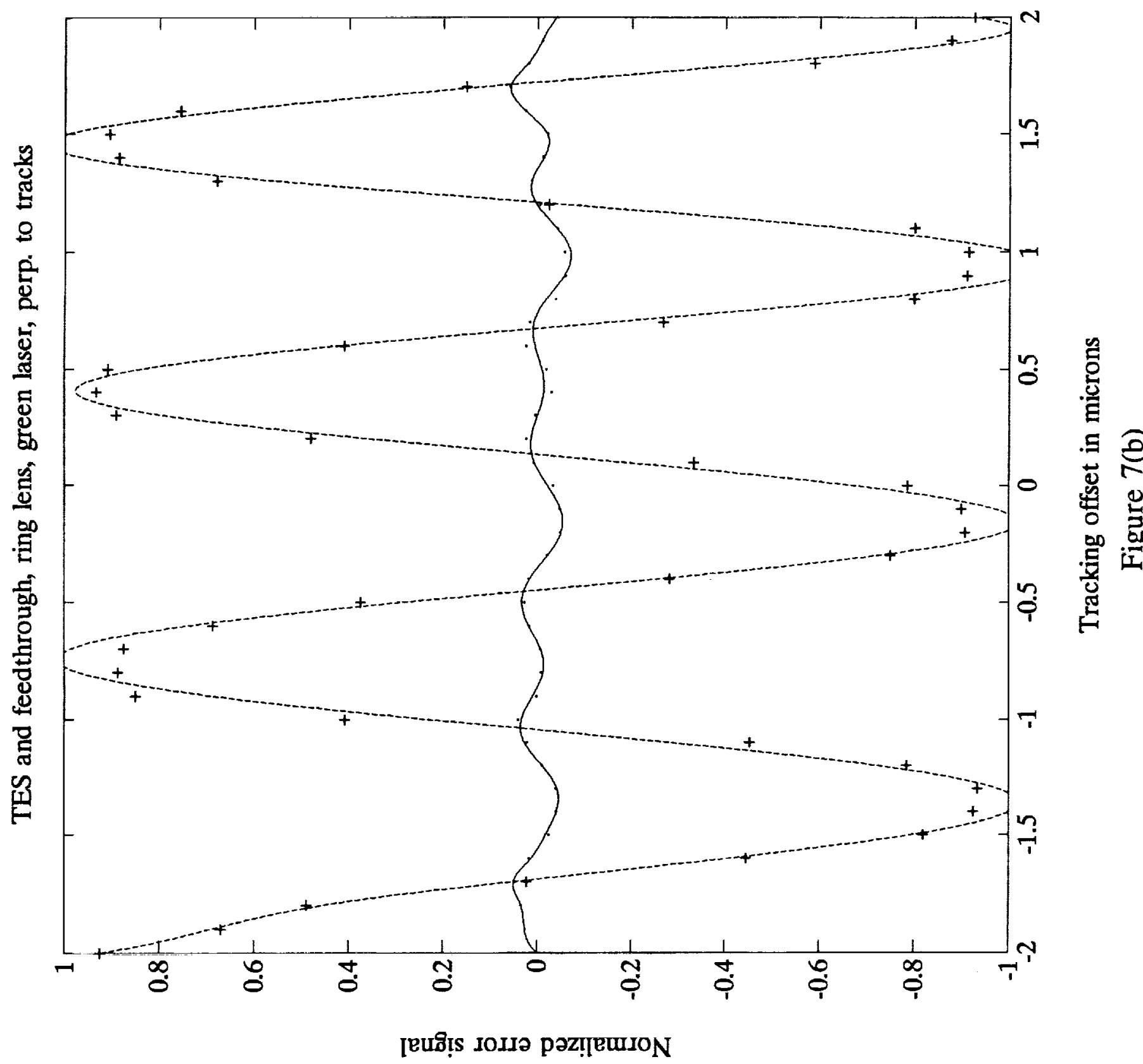




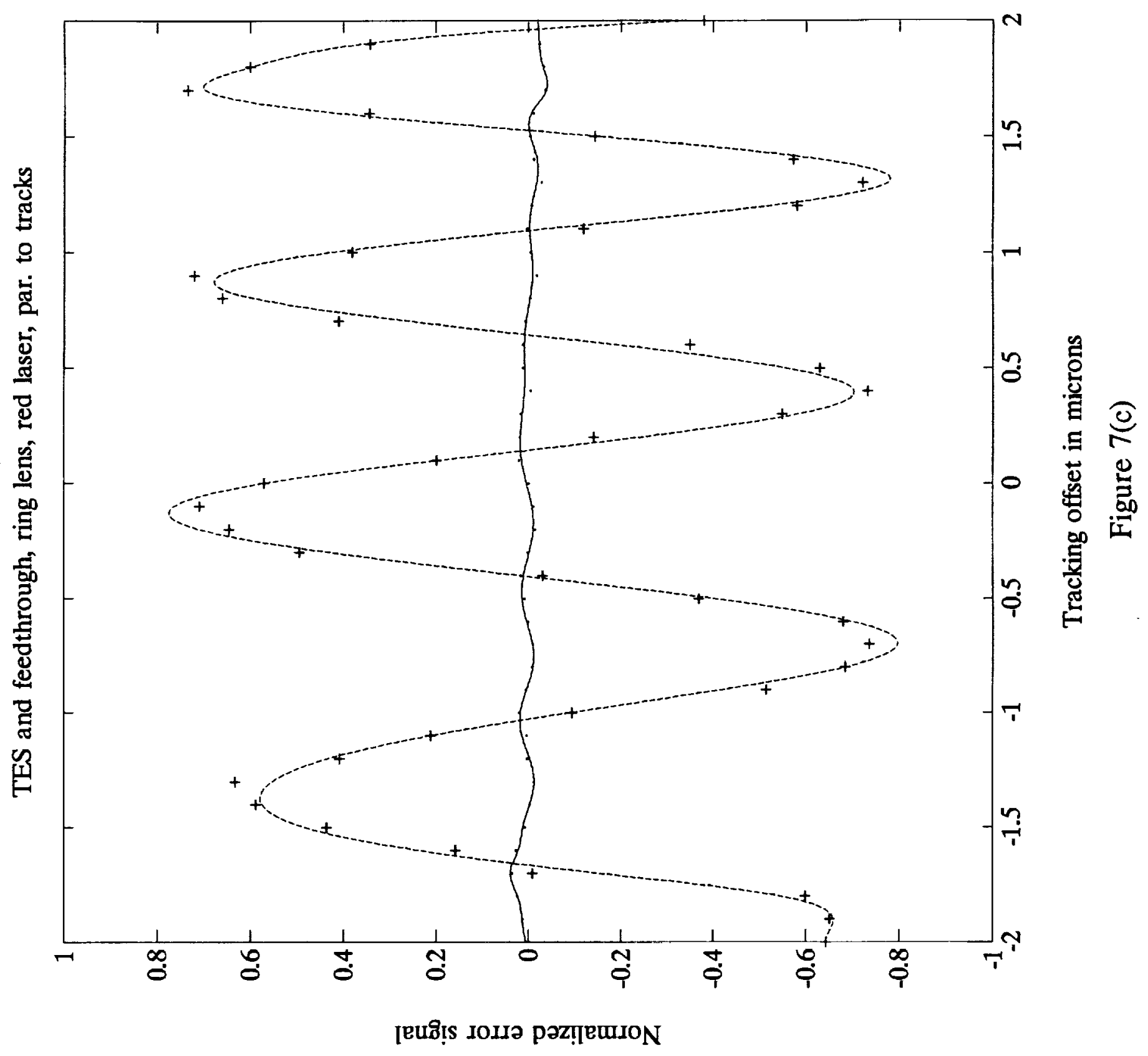




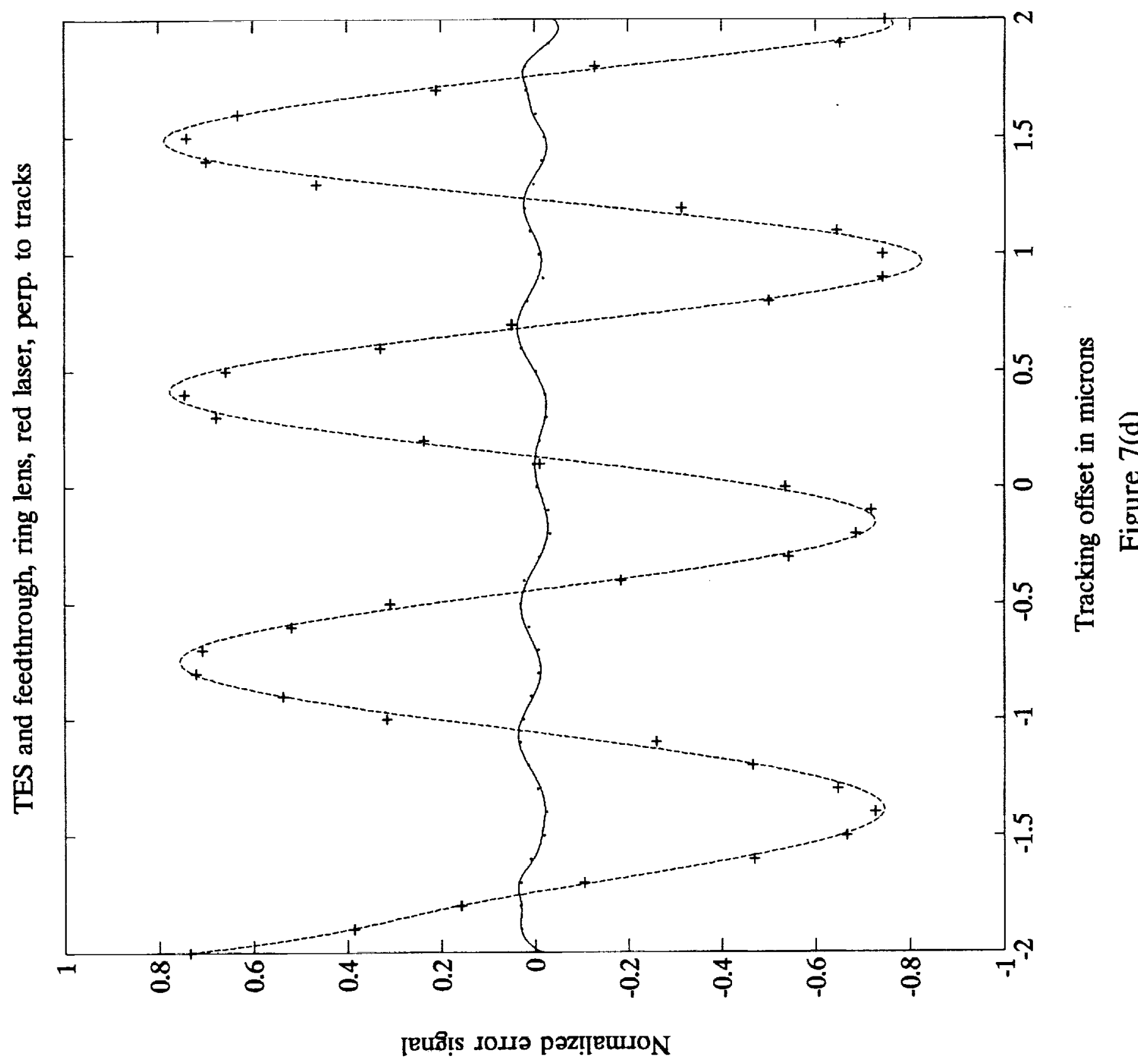




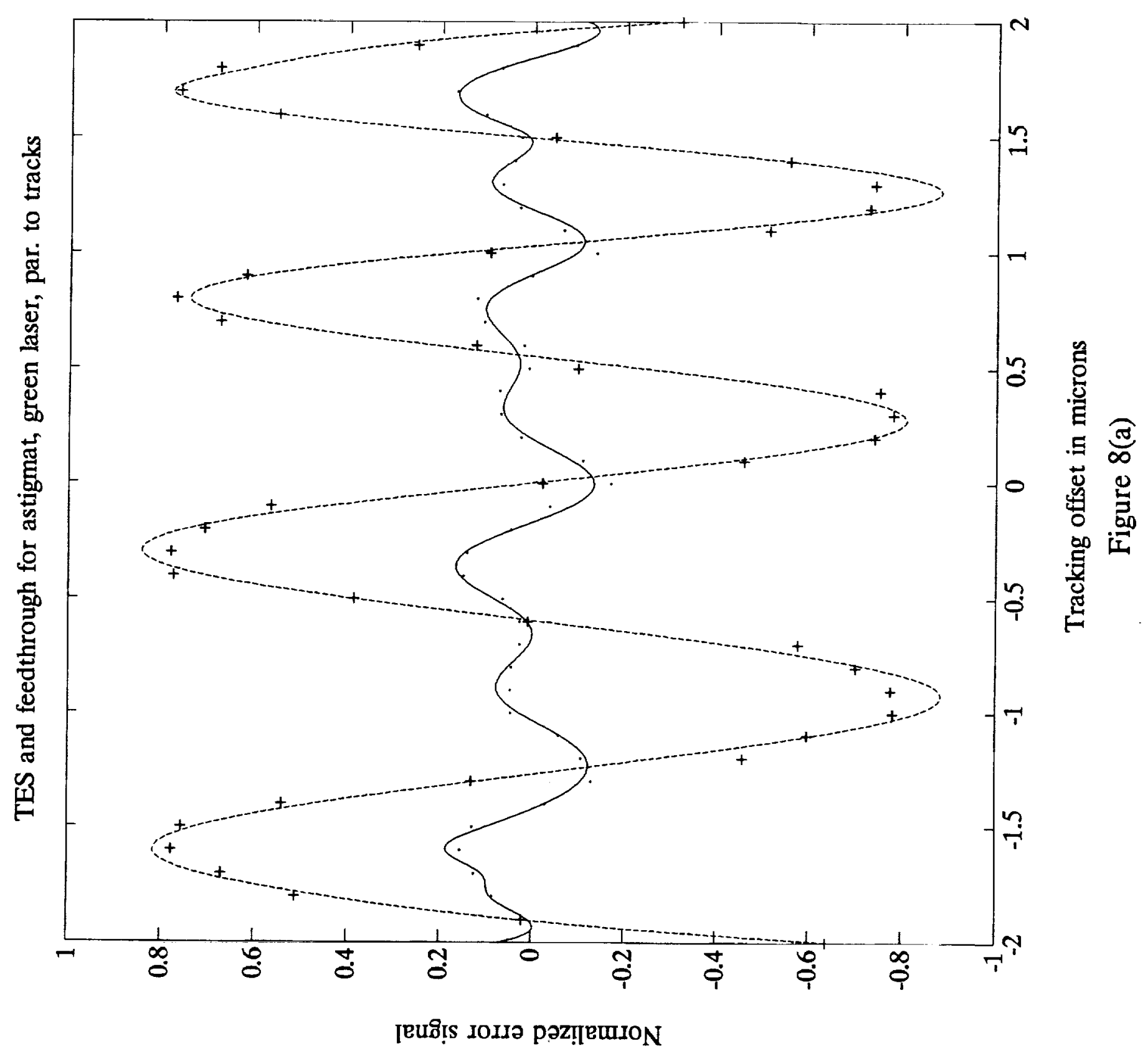




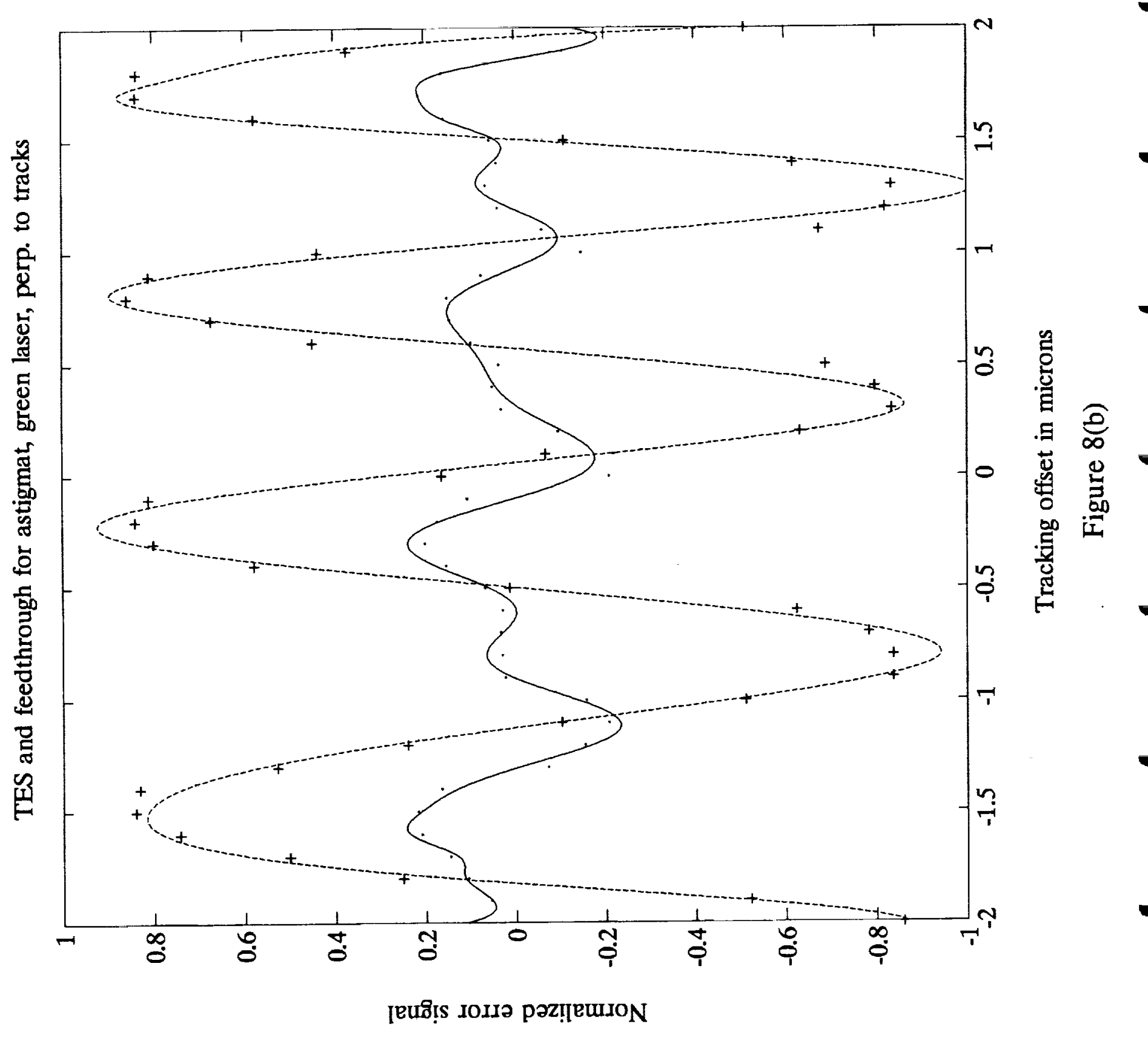




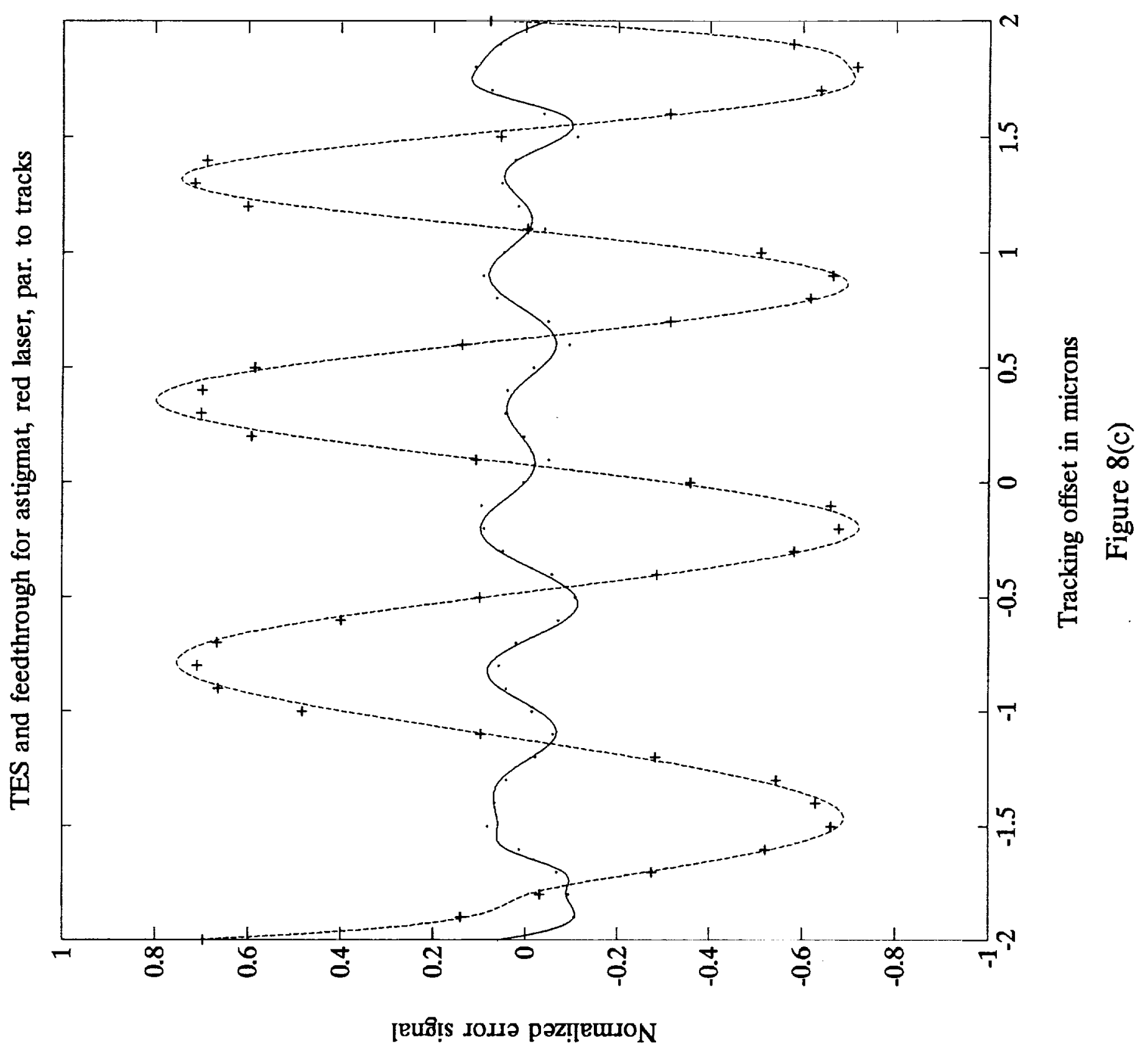




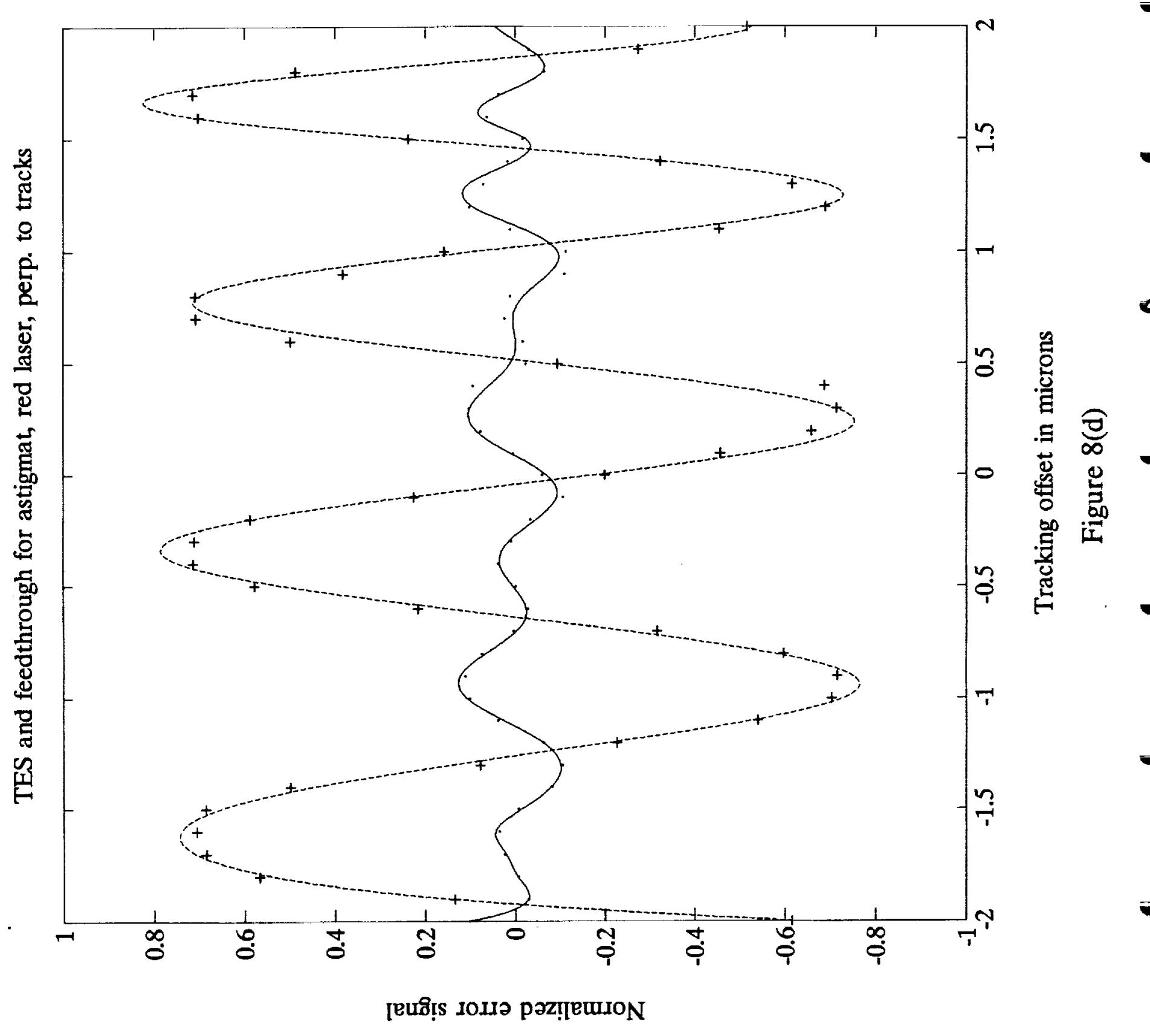




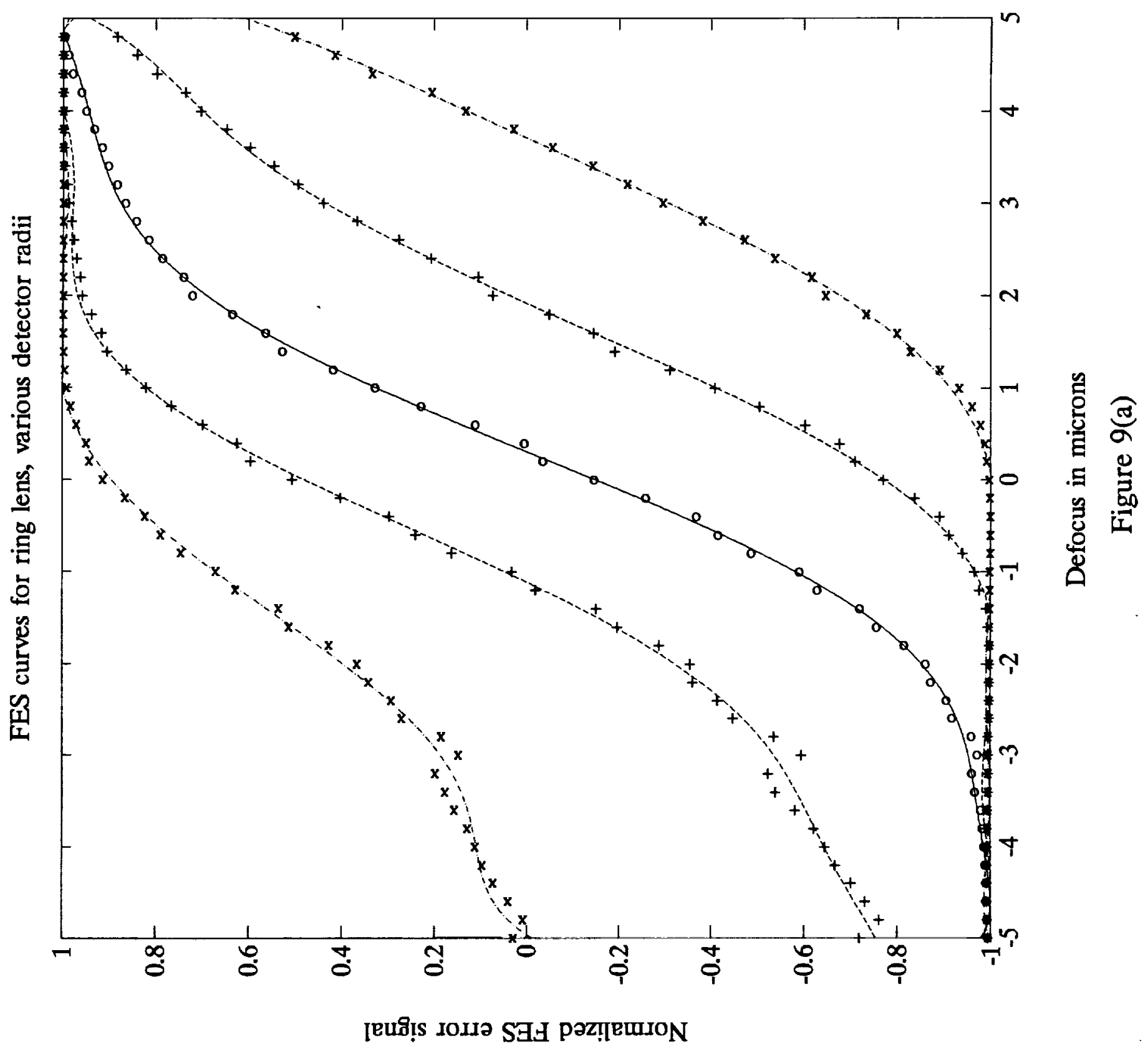




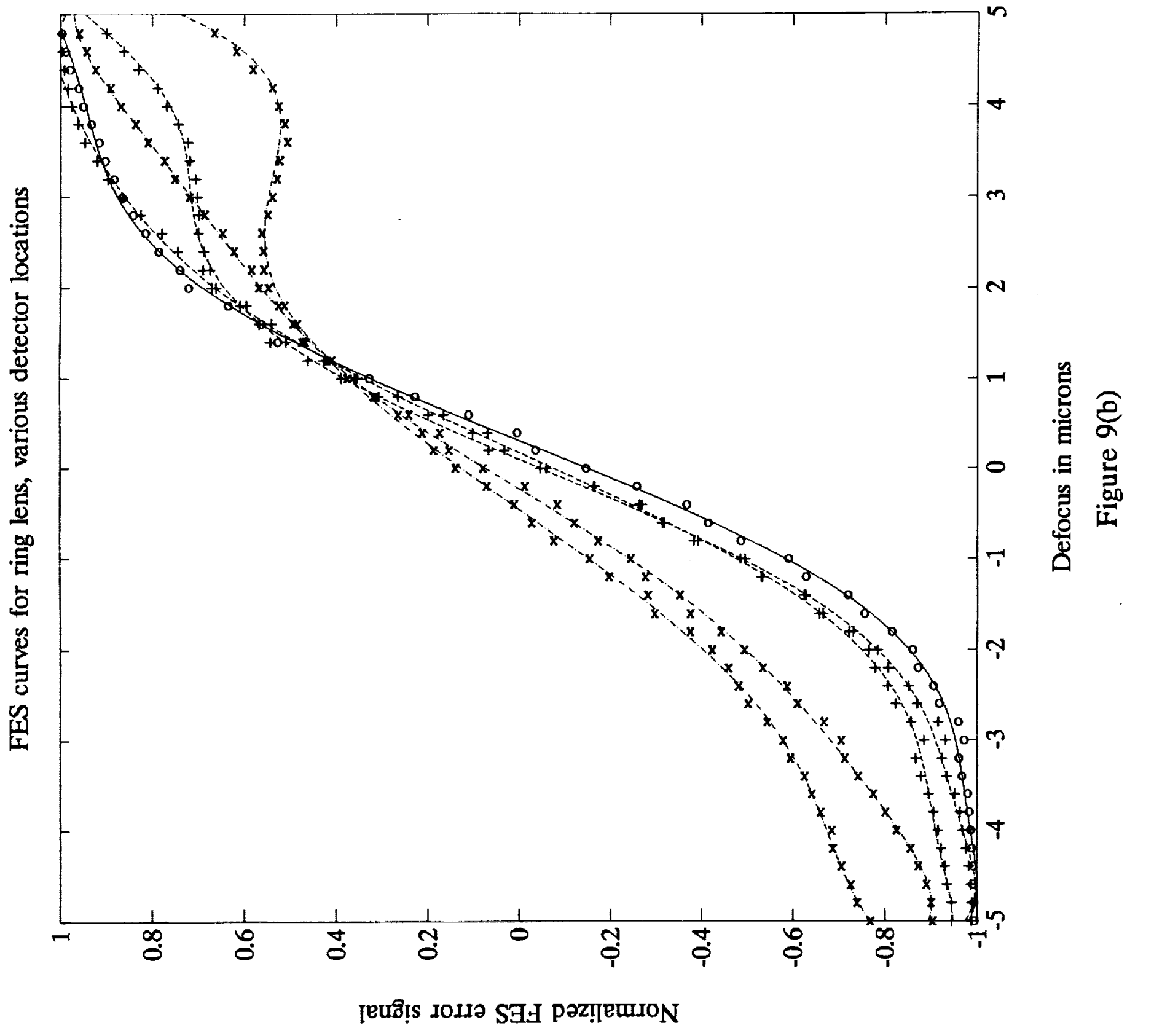




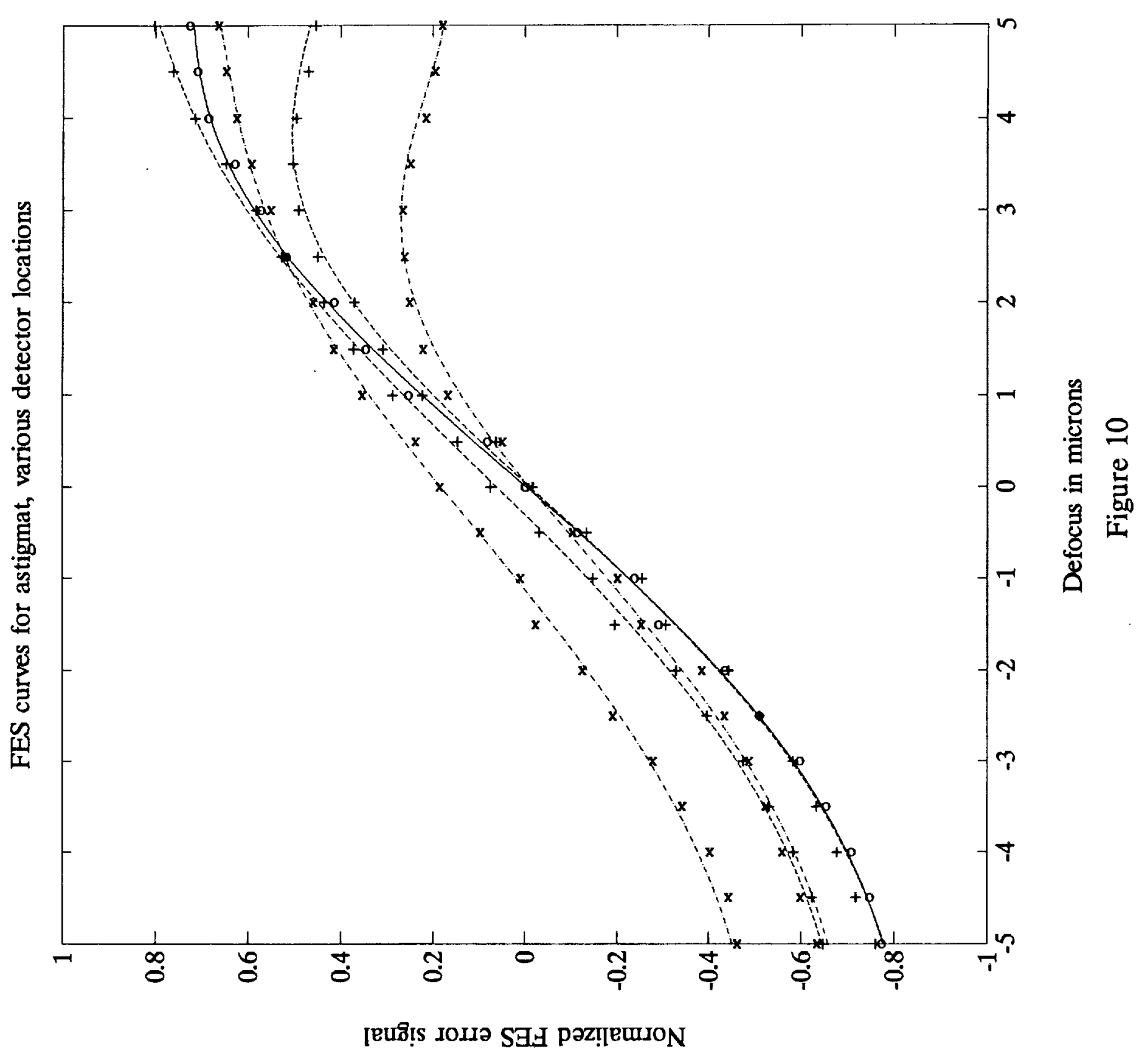


$\sigma$

$\nabla$

$\sigma$

$=$

$\nabla$

$-$

$\sigma$ 


\section{APPENDIX C}


$-$

$\nabla$ $\bullet$

$=$

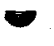

